\title{
Photosynthetic Characteristics of Chinese Cabbage Sprayed with Indoleacetic Acid under Aluminum Stress
}

\author{
Huaqiang $\operatorname{Tan}^{1, \mathrm{a}}$, Jianjun Yan ${ }^{2, \mathrm{~b}}$ and Huanxiu $\mathrm{Li}^{1, \mathrm{c} \text { * }}$ \\ ${ }^{1}$ Institute of Pomology and Olericulture, Sichuan Agricultural University, Chengdu, Sichuan, China \\ ${ }^{2}$ College of Horticulture, Sichuan Agricultural University, Chengdu, Sichuan, China \\ a307927595@qq.com, b1139522849@qq.com, chxli62@163.com \\ *Corresponding author
}

Keywords: Indoleacetic acid; Photosynthetic characteristics; Chinese cabbage

Abstract. A pot experiment was conducted to study the effects of exogenous indoleacetic acid (IAA) on photosynthetic characteristics of Chinese cabbage under aluminum (Al) stress. Five treatments were used in the experiment: leaves were sprayed with $0(\mathrm{CK}), 50,100,150$ and $200 \mu \mathrm{mol} \cdot \mathrm{L}^{-1}$ concentrations of indoleacetic acid solution. The results showed that indoleacetic acid can improve the adaptability and photosynthetic capacity of Chinese cabbage seedlings under Al stress. With the increasing of indoleacetic acid concentration, net photosynthetic rate (Pn) of Chinese cabbage were enhanced significantly. The result of transpiration rate (Tr), light use efficiency (LUE), water use efficiency (WUE) and stomatal conductance (Gs) were the same as Pn. Treatments of different concentrations of indoleacetic acid on Chinese cabbage under aluminum stress has not significant effect on $\mathrm{CO}_{2}$ concentration of intercellular (Ci). Therefore, indoleacetic acid could use to enhance the photosynthetic ability of Chinese cabbage, which would help to improve the adaptability of Chinese cabbage, and the best concentration was $150 \mu \mathrm{mol} \cdot \mathrm{L}^{-1}$.

\section{Introduction}

Aluminum is the most abundant metal element in the earth crust. Under acidic conditions $(\mathrm{pH}<5)$, insoluble aluminum will become soluble aluminum in soil, and cause harm to plants, hampered the growth of plants seriously $[1,2]$. With the aggravation of soil acidification, aluminum has become an important factor inhibiting plant growth in acid soils [3].

Auxin is the first discovery of a class of plant hormone promoting plant growth, and indoleacetic acid is one of the most important auxin[4,5]. Studies have shown that exogenous IAA can effectively alleviate the toxic effects of aluminum on plants [6,7].

Chinese cabbage (Brassica rapa ssp. pekinensis) is rich in nutrients, which is preferred by consumers in China [8]. In this study, we used different concentrations of indoleacetic acid to spray Chinese cabbage seedlings, to investigate the influence of indoleacetic acid on photosynthetic characteristics of Chinese cabbage in aluminum stress.

\section{Materials and Methods}

Materials. The experiments were conducted at Sichuan Agricultural University $\left(30^{\circ} 42^{\prime} \mathrm{N}, 103^{\circ} 51^{\prime}\right.$ E), Wenjiang, China. The seeds of Chinese cabbage named quick 35 were harvested in 2014 and purchased from Chengdu, China. All chemicals used in experiments were of analytical grade.

Experimental Design. Seeds were sterilized in 10\% sodium phosphate solution for 30 minutes, flushed five times in distilled water, and then placed on 9-cm-diameter Petri dishes with three layers of filter paper moistened with distilled water and germinated at $25^{\circ} \mathrm{C}$ in darkness. Seeds were considered germinated when the seed coat was broken and a radicle was visible. After germination, seeds were planted in nutrition pot filled with vermiculite and perlite, the pot was ten centimeters in diameter and height.

Seedlings were irrigated with $20 \mathrm{ml}$ Hoagland nutrient solution containing $50 \mu \mathrm{mol} \cdot \mathrm{L}^{-1}$ concentrations of aluminum every other day, until the experiment finishing. 
When the third leaf expanded, their leaves were sprayed with $0(\mathrm{CK}), 50,100,150,200 \mu \mathrm{mol} \cdot \mathrm{L}^{-1}$ concentrations of indoleacetic acid solution until foliage and dorsal dripping. Seedlings were sprayed with indoleacetic acid solution every other day, and three times in total. Each treatment consisted of 10 pots with one plant per pot. Positions of the pots were randomly changed daily to minimize positional effects. 30 days after treatment, the photosynthesis of each plant was determined by using LI-6400 portable photosynthesis meter (LI-COR Inc., USA). The photosynthetic parameters of the photosynthesis meter were manual control $\mathrm{CO}_{2}$ concentration $400 \mu \mathrm{mol} \cdot \mathrm{CO}_{2} \mathrm{~mol}^{-1}$, temperature $25^{\circ} \mathrm{C}$, light intensity $1200 \mu \mathrm{mol} \mathrm{m} \mathrm{m}^{-2} \cdot \mathrm{s}^{-1}$. The determination of photosynthetic parameters were net photosynthetic rate $(\mathrm{Pn})$, transpiration rate $(\mathrm{Tr})$, stomatal conductance $(\mathrm{Gs})$ and $\mathrm{CO}_{2}$ concentration of intercellular $(\mathrm{Ci})$, and each treatment was repeated three times. Water use efficiency $($ WUE$)=$ net photosynthetic rate $(\mathrm{Pn}) /$ transpiration rate $(\mathrm{Tr})$, Light use efficiency $(\mathrm{LUE})=$ net photosynthetic rate (Pn) / light intensity[9].

Statistic analyses. Statistical analyses were performed using SPSS 13.0 statistical software (IBM, Chicago, IL, USA). Data were analyzed by one-way ANOVA with least significant difference (LSD) at a $5 \%$ confidence level.

\section{Results and Discussion}

Net Photosynthetic Rate (Pn). Indoleacetic acid enhanced Pn of Chinese cabbage under Al stress significantly,compared with CK. And the Pn of Chinese cabbage was increased with the increasing of indoleacetic acid concentration from $50 \mu \mathrm{mol} \cdot \mathrm{L}^{-1}$ up to $150 \mu \mathrm{mol} \cdot \mathrm{L}^{-1}$ (Fig. 1). Compared with CK, when the concentration of indoleacetic acid were 50, 100 and $200 \mu \mathrm{mol} \cdot \mathrm{L}^{-1}$, these treatments enhanced Pn of Chinese cabbage by $16.94 \%(p>0.05), 35.07 \%(p<0.05)$ and $17.74 \%(p>0.05)$ respectively. When the concentration of indoleacetic acid was $150 \mu \mathrm{mol} \cdot \mathrm{L}^{-1}$, Pn of Chinese cabbage reached the highest, and increased by $45.83 \%(p<0.05)$. In this research, it was illustrated that indoleacetic acid can improve photosynthetic capacity of Chinese cabbage seedlings, and alleviate the Al stress.

Transpiration Rate (Tr). Indoleacetic acid increased the Tr of Chinese cabbage compared with CK (Fig. 2). The trend of $\operatorname{Tr}$ was consistent with Pn. Compared with CK, when the concentration of indoleacetic acid was $150 \mu \mathrm{mol} \cdot \mathrm{L}^{-1}$, $\operatorname{Tr}$ of Chinese cabbage reached the highest, and increased by $45.30 \%(p<0.05)$. When the concentration of indoleacetic acid were 50,100 and $200 \mu \mathrm{mol} \cdot \mathrm{L}^{-1}$, these treatments enhanced Tr of Chinese cabbage by 5.82\% $(p>0.05), 23.30 \%(p<0.05)$ and $24.72 \%(p<$ $0.05)$ respectively. It was showed that indoleacetic acid can alleviate the Al stress of Chinese cabbage seedlings.

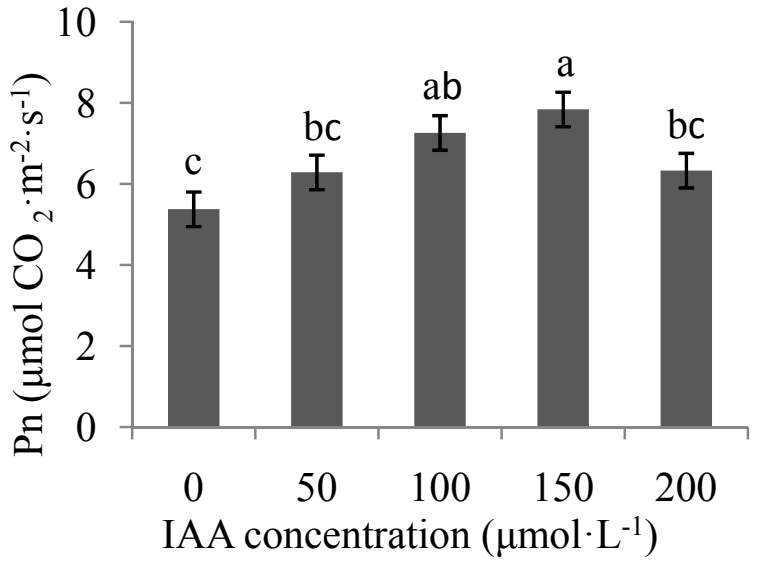

Fig. 1 Pn of IAA sprayed Chinese cabbage

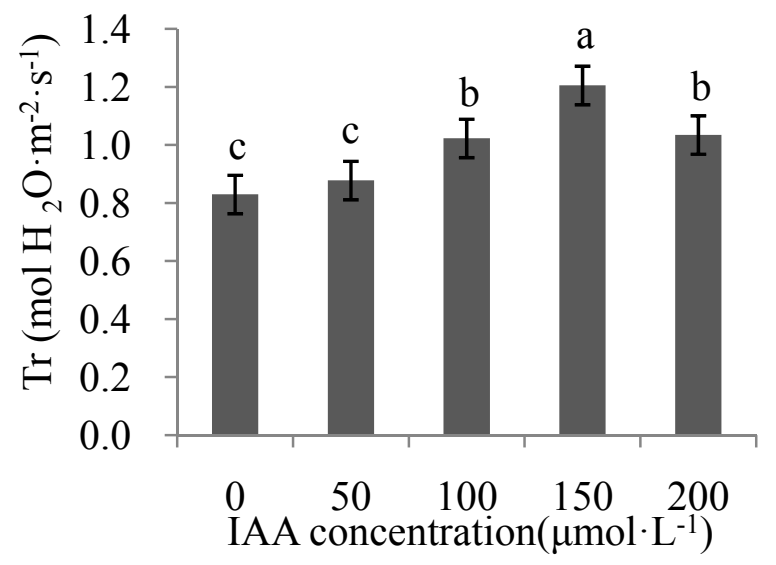

Fig. 2 Tr of IAA sprayed Chinese cabbage 
Water Use Efficiency (WUE). The difference between WUE of Chinese cabbage treated with different concentrations of indoleacetic acid under Al stress were not significant. It is illustrated that spraying indoleacetic acid has no obvious effect on WUE of Chinese cabbage under Al stress, compared with CK.

Light Use Efficiency (LUE). Indoleacetic acid increased the LUE of Chinese cabbage with the increasing of indoleacetic acid concentration under Al stress (Fig. 4). When the concentration of indoleacetic acid were 50,100 and $200 \mu \mathrm{mol} \cdot \mathrm{L}^{-1}$, these treatments enhanced LUE of Chinese cabbage by $16.94 \%(p>0.05), 35.07 \%(p<0.05)$ and $17.74 \%(p>0.05)$ respectively, compared with CK. When the concentration of indoleacetic acid was $150 \mu \mathrm{mol} \cdot \mathrm{L}^{-1}$, LUE of Chinese cabbage reached the highest, and increased by $45.83 \%(p<0.05)$. It was showed that indoleacetic acid can alleviate the Al stress of Chinese cabbage seedlings.

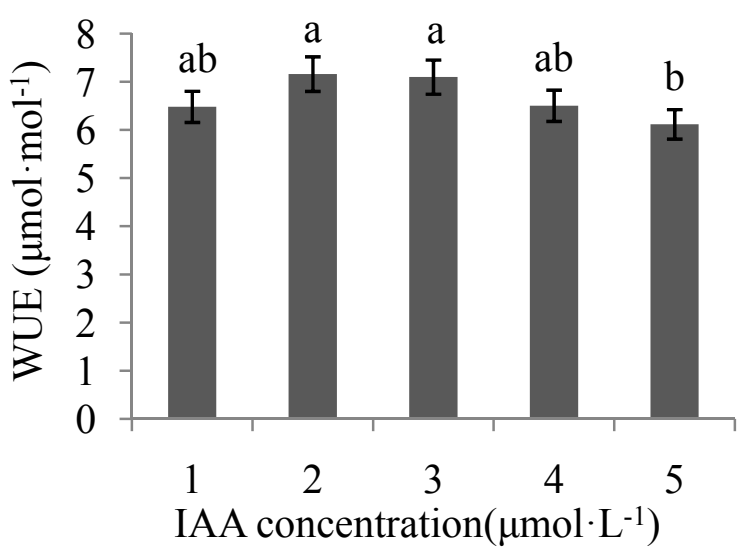

Fig. 3 WUE of IAA sprayed Chinese cabbage

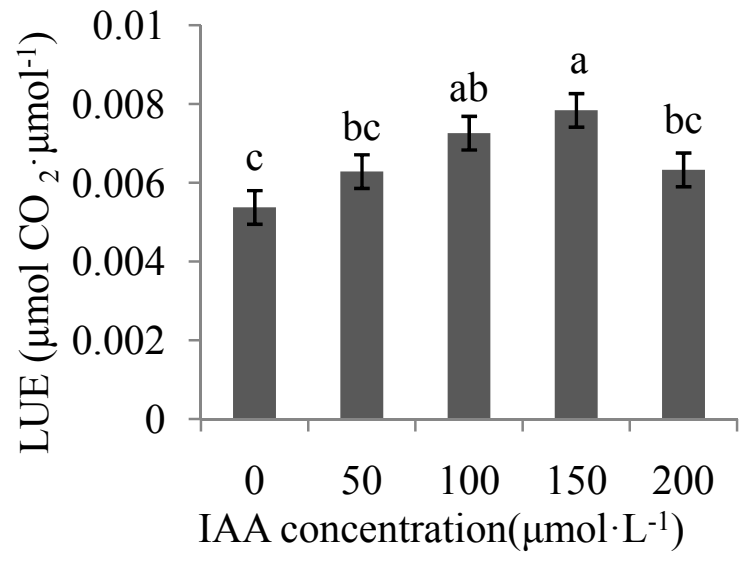

Fig. 4 LUE of IAA sprayed Chinese cabbage

Stomatal Conductance (Gs). After spraying indoleacetic acid, Gs of Chinese cabbage increased (Fig. 5). The Gs of Chinese cabbage was increased with the increasing of indoleacetic acid concentration from $50 \mu \mathrm{mol} \cdot \mathrm{L}^{-1}$ up to $150 \mu \mathrm{mol} \cdot \mathrm{L}^{-1}$. Gs of Chinese cabbage reached the highest, and increased by $52.43 \%(p<0.05)$ compared with $C K$. When the concentration of indoleacetic acid were 50, 100 and $200 \mu \mathrm{mol} \cdot \mathrm{L}^{-1}$, these treatments enhanced Gs of Chinese cabbage by $5.13 \%(p>0.05), 29.47 \%(p<$ $0.05)$ and $24.46 \%(p<0.05)$ respectively.

$\mathrm{CO}_{2}$ Concentration of Intercellular (Ci). The Fig. 6 shows that Ci of Chinese cabbage decreased by indoleacetic acid treatment under $\mathrm{Al}$ stress, and the difference between treatments were not significant. It is showed that the effect on $\mathrm{Ci}$ of indoleacetic acid was not obvious.

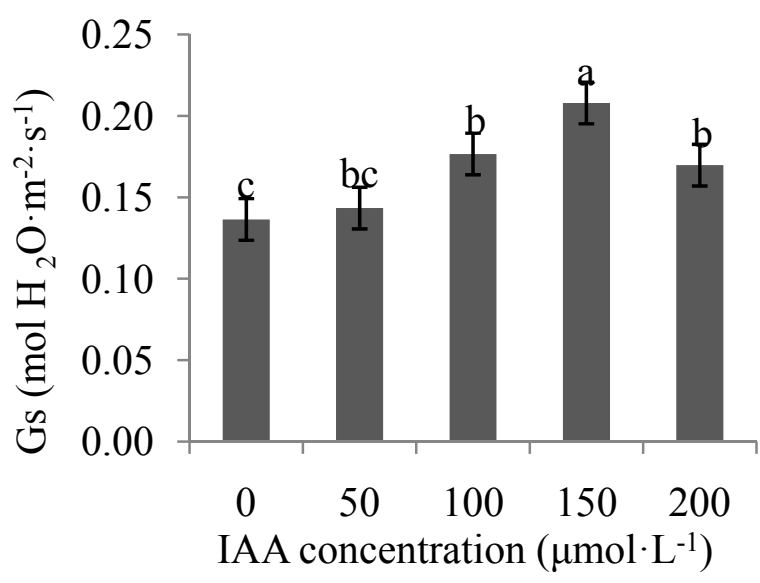

Fig. 5 Gs of IAA sprayed Chinese cabbage

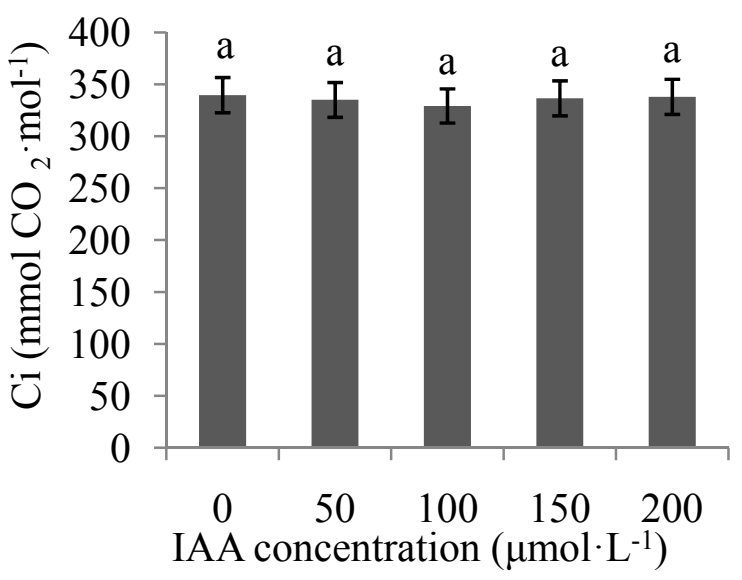

Fig. $6 \mathrm{Ci}$ of IAA sprayed Chinese cabbage 


\section{Conclusions}

Indoleacetic acid can improve the adaptability and photosynthetic capacity of Chinese cabbage seedlings under Al stress. With the increasing of indoleacetic acid concentration, Pn of Chinese cabbage were enhanced significantly. The result of Tr, LUE, WUE and Gs were the same as Pn. Treatments of different concentrations of indoleacetic acid on Chinese cabbage under aluminum stress has not significant effect on $\mathrm{Ci}$. Therefore, indoleacetic acid could use to enhance the photosynthetic ability of Chinese cabbage.

\section{Acknowledgements}

This work was financially supported by the Sichuan Agricultural University "Shuang-Zhi Plan" Foundation.

\section{References}

[1] J.H. Weng, L.F. Huang and X.R. Liu: China Environmental Science Vol. 20 (2000), p.501-505 (In Chinese).

[2] Q. Liu and W.W. Long: Journal of Jinggangshan University(Natural Sciences Edition) Vol. 03 (2011), p.56-59 (In Chinese).

[3] C.D. Foy and M.S. White: Annual Review of Plant Physiology Vol. 29 (1978), p.511-566.

[4] J. Li and J.Z. Cui: Biotechnology Bulletin Vol. 06 (2012), p.13-17 (In Chinese).

[5] J.L. Wang and A.M. Zhang: Bulletin of Botany Vol. 03 (2012), p.292-301 (In Chinese).

[6] P. Wang, D.J. Chen and K.Z. Li: Acta Botanica Boreali-Occidentalia Sinica Vol. 01 (2014), p.112-117 (In Chinese).

[7] X.F. Ying and P. Liu: Chinese Journal of Applied Ecology Vol. 16 (2005), p.166-170 (In Chinese).

[8] J. Zhu: Hubei Agricultural Sciences Vol. 19 (2012), p.4278-4283 (In Chinese).

[9] X.J. Jiang, H. Wang and W. Peng: Shanxi Journal of Agricultural Sciences Vol. 54 (2008), p.56-58 (In Chinese). 